\title{
Traumatic asymmetrical bilateral hip dislocation: A rare case report
}

\author{
Weicheng Qin, MD(D, Yue Fang, MD (D) \\ West China Hospital of Sichuan University, Orthopaedic Institute, Chengdu, China
}

The hip joint is an inherently stable joint, with a significant force being required for dislocation to take place. ${ }^{[1]}$ Dislocation of the hip presents an extremely low proportion of all joint dislocation. In the literature, asymmetrical bilateral hip dislocation and bilateral dislocation account for 2 to $5 \%$ of joint dislocations of any nature and for only $1 \%$ of all hip dislocations..$^{[1-3]}$

In this article, we present a rare case of asymmetrical bilateral hip dislocations combined with acetabulum and bilateral pubis fractures. In addition to other forms of hip dislocation, the morbidity rate of such a case is quite low. Relevant literature reveals no record of similar cases with a corresponding injury mechanism.

\section{CASE REPORT}

A 49-year-old male was working at a mine unrestrained was involved in a serious tramcar accident. He was admitted to the local emergency department with an open injury of the left lower limb, bilateral hip

Received: June 06, 2021

Accepted: June 28, 2021

Published online: November 19, 2021

Correspondence: Yue Fang, MD. West China Hospital of Sichuan University, Orthopaedic Institute, 610044 Chengdu, China.

E-mail: fangyue1968@163.com

Doi: $10.52312 / j d r s .2021 .290$

Citation: Qin W, Fang Y. Traumatic asymmetrical bilateral hip dislocation: A rare case report. Jt Dis Relat Surg 2021;32(3):767-770.

○2021 All right reserved by the Turkish Joint Diseases Foundation

This is an open access article under the terms of the Creative Commons Attribution-NonCommercial License, which permits use, distribution and reproduction in any medium, provided the original work is properly cited and is not used for commercial purposes (http://creativecommons.org/licenses/by-nc/4.0/).

\section{ABSTRACT}

Traumatic bilateral hip dislocation is a rare phenomenon. Simultaneous traumatic asymmetrical bilateral hip dislocation is even more unusual. There is no report in the literature regarding the mechanism of injury in tramcar squeezing. A 49-year-old male who had a serious tramcar accident and developed asymmetric bilateral hip dislocations (left posterior, right anterior) combined with fracture of left acetabulum and multiple injuries was admitted. Right hip dislocation was treated with manual reduction. Left hip dislocation, fracture of acetabulum and bilateral pubis, and open wound of left thigh were treated with open reduction and internal fixation. In conclusion, hip dislocation should be performed with manual reduction within $6 \mathrm{~h}$. If manual reduction fails, open reduction and even internal fixation can be performed to prevent complications, such as avascular necrosis of the femoral and heterotopic ossification.

Keywords: Asymmetrical hip dislocation, trauma, traumatic hip dislocation.

pain and deformity combined with and abdominal injury $5 \mathrm{~h}$ after the accident. Bilateral supracondylar traction of the femur and debridement and suture of the left thigh were performed immediately at the local hospital. Due to the concomitant visceral injury, an exploratory laparotomy was performed and, one day later, the patient was transferred to the emergency department of our hospital after hemodynamic stability was achieved.

In our hospital, the left lower extremity was adducted, flexed, and internally rotated, with $20 \mathrm{~cm}$ infectious wound on the back of his thigh. The right lower extremity was abducted, flexed and externally rotated (Figure 1). In the emergency department, pelvis computed tomography (CT) revealed asymmetrical bilateral hip dislocation (left posterior, right anterior) and fractures of the left acetabulum and bilateral pubis (Figure 2). Other injures included fractures lumbar 1-5 transverse processes, liver, kidney, and lung dysfunction. The patient was transferred to 
the operating room where manual reduction was performed under general anesthesia. The anterior dislocation of the right hip joint was successfully reduced. Manual reduction of the posterior dislocation of the left hip joint was also attempted; however, reduction failed after the second attempt. Due to hemodynamic instability, manual reduction was not attempted and open reduction was not considered. Meanwhile, the patient was transferred to the monitoring room of the trauma center to maintain its hemodynamic stability. Postoperative three-dimensional CT scan showed that the right femoral head was in place and the left femoral head was located behind and above the acetabulum. After the evaluation of the anesthesiology department, emergency operation was performed on the second day. Open reduction and internal fixation of the left acetabular posterior wall were performed combined with open reduction of the left hip joint and debridement of the left thigh.

After surgery, deformities of both lower limbs were corrected. Due to severe systemic symptoms and multiple injuries, the patient was transferred to the surgical intensive care unit for the supportive treatment. Postoperative CT scan revealed that

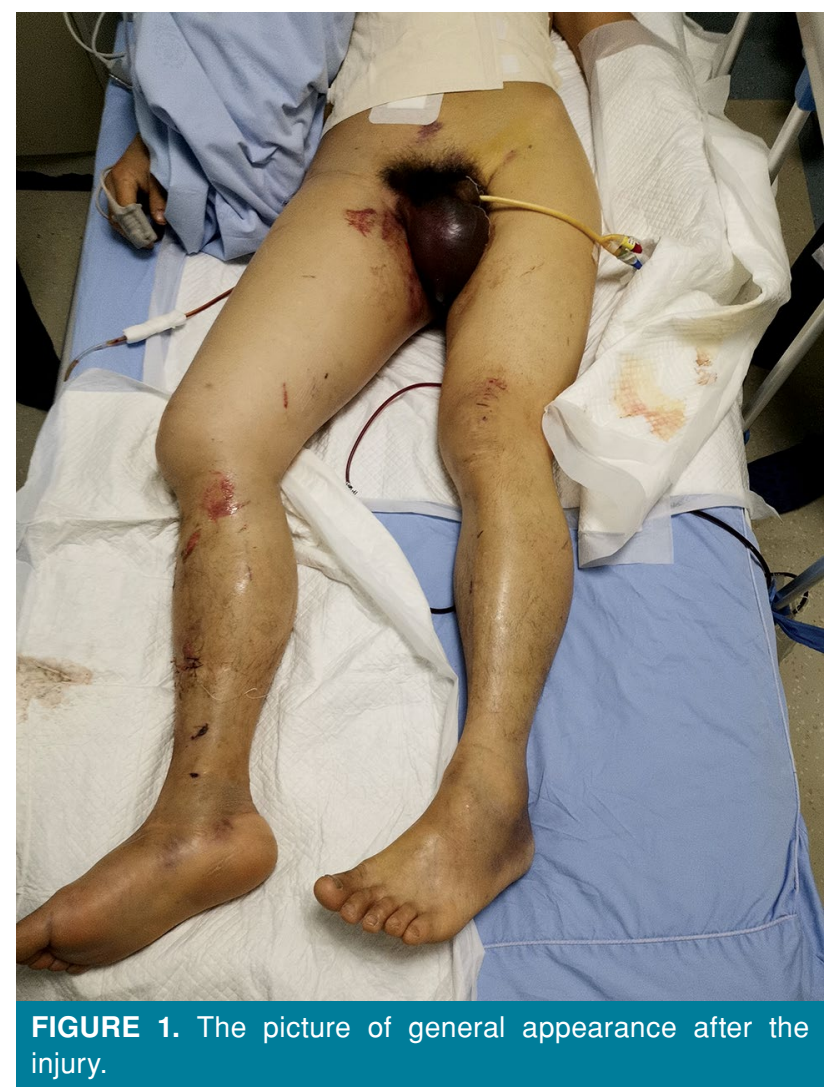

the fracture fragment in the posterior wall of left acetabular was successfully reduced. Three weeks after the injury, the left acetabular anterior wall fracture was treated with open reduction and internal fixation (Figure 3). After the operation, the patient received physical therapy in bed including quadriceps strengthening exercises and active range of motion. The left hip pain was relieved successfully after surgery.
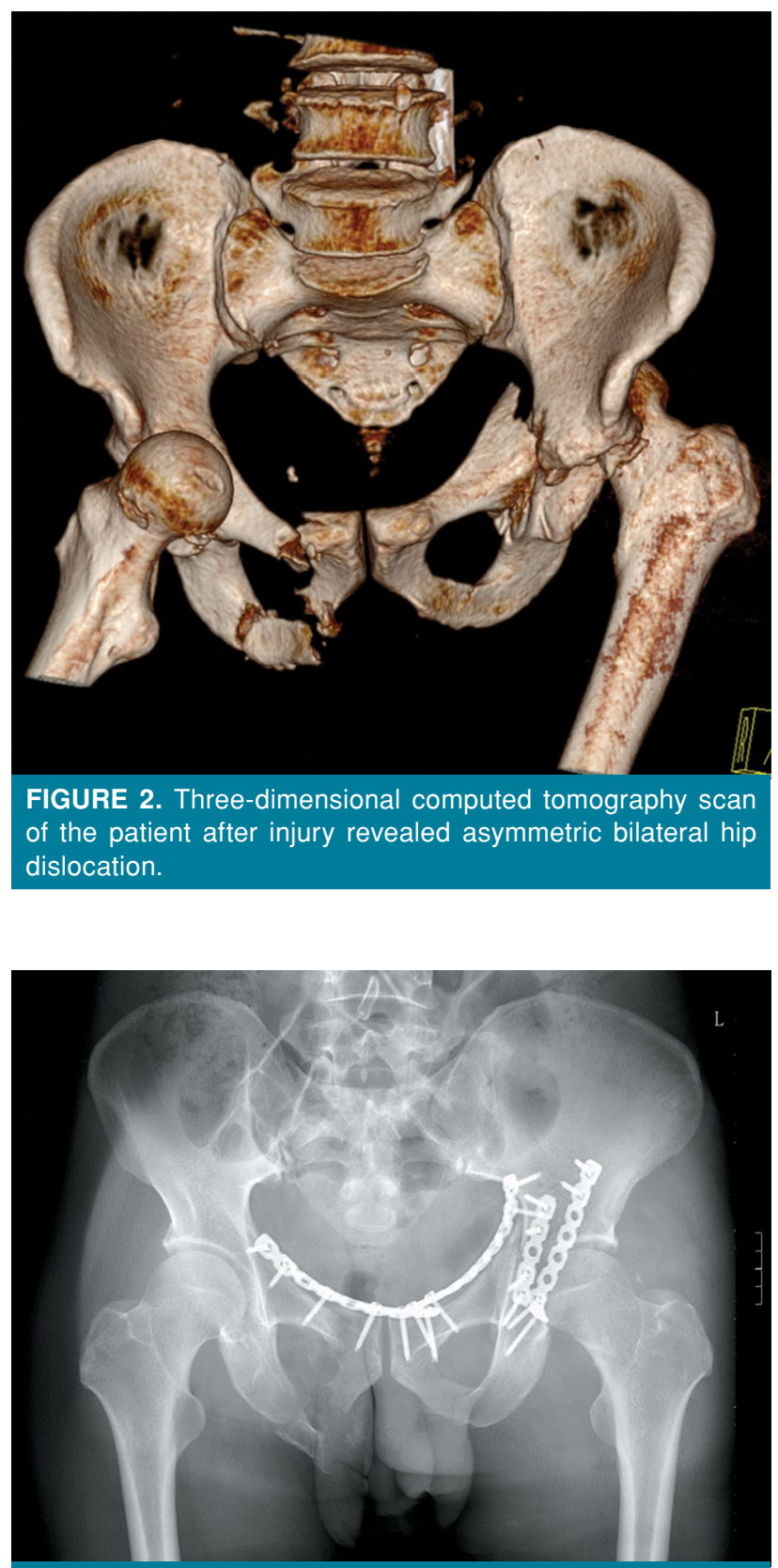

FIGURE 3. Postoperative anteroposterior radiograph of the pelvis showing bilateral hip joints in place and good reduction of acetabular fractures. 
Five months after the injury, the patient was usually in a good condition. The quadriceps muscle strength of both lower limbs at level $\mathrm{V}$, the posterior muscle group of the lower leg at level IV+, and the anterior muscle group at level IV+ with a good range of motion was achieved. The flexion of the left knee joint was slightly impaired due to tissue necrosis at the back of the left thigh and partial removal of the biceps femoris. Radiological evaluation showed that both hips, pelvis, and acetabulum were in a good shape. However, heterotopic ossification of the left hip joint was observed. No osteonecrosis of the femoral head in bilateral hip joints was observed. The patient was lost-to-follow-up due to noncompliance to scheduled visits. A written informed consent was obtained from the patient.

\section{DISCUSSION}

Traumatic hip dislocations account for 2 to 5\% of all dislocation, while bilateral hip dislocation accounts for $1 \%$ of all hip dislocations. Hip dislocation is usually common in traffic accidents, but asymmetrical bilateral hip dislocation is rare. ${ }^{[4,5]}$ We reviewed the literature reports associated with asymmetrical bilateral hip dislocation. The most common injury mechanism in the English literatures is "instrument panel injury". ${ }^{[5-12]}$ The second most common mode of injury was weight from above $(n=17,19 \%)$, followed by pedestrian struck by motor vehicle $(\mathrm{n}=14,15 \%)$ and fall $(n=5,5 \%) \cdot{ }^{[10]}$ Currently, it is mainly believed that the type of traumatic hip dislocation is determined by the position of the lower limb at the time of the impact and the direction of the vector applied. The force acts on the knee relative to the adduction of the hip joint, creating a backward force, resulting in posterior dislocation. The force acts on the knee relative to the abduction and rotation of the hip joint, creating a forward force, leading to anterior dislocation. ${ }^{[6]}$ In posterior dislocation, the hip rotates inward, bends and adducts, while the hip rotates outward and abducts in anterior dislocation. ${ }^{[7]}$ In the present case, the tramcar hit the lumbosacral area from the left and back of the patient, causing the body to rotate to the right and flexion of the hips and knees, and flexion of the waist. The right femoral head was displaced downward and forward relative to the acetabulum, and the left femoral head was displaced backward and forward relative to the acetabulum. The tramcar, then, pushed the patient to the wall and both knees hit the wall, causing asymmetrical bilateral hip dislocation (Figure 4).

Fang $^{[13]}$ reported a case of interesting and mild similar injury mechanism. If a heavy object falls on the lumbosacral region of a person who is bending down, the lower extremity is in a posture of hip and knee flexion, the pressure on the lumbosacral area increases and causes the head of the femur head to move backward, leading to posterior dislocation. Meanwhile, the hip joint that is further back is in extension, and the increased force in the lumbosacral area causes anterior dislocation of the hip joint. This kind of dislocation may also occur, if one of the hip joints is flexed and adducted, and the other abducted and flexed during the process of the injury. ${ }^{[13]}$ In our case, the movement of the hip joint was restricted after manual reduction, and the T-shaped pillow was used to maintain abduction and external rotation. Abdulfattah Abdullah et al. ${ }^{[14]}$ reported that the patient should rest in bed for three weeks, and use an T-shaped pillow, then remove the traction and start physical therapy. Karaarslan et al. ${ }^{[15]}$ concluded that successful treatment, early full-load mobilization, and early passive motion plus active auxiliary motion were very useful.

Once associated injuries are ruled out, reduction should be performed as soon as possible under general anesthesia or conscious sedation. If full muscular sedation is available in a safe manner in the emergency room and there are no other contraindications to attempted reduction; e.g., femoral neck fracture or acetabulum fracture, and reduction can be performed in a safe and timely manner in the emergency room setting. ${ }^{[10]}$ The current main view is that hip dislocation should be reduction within $6 \mathrm{~h}$ as far as possible, and at least within $24 \mathrm{~h}$. The earlier reduction, the risk of avascular necrosis of the femoral

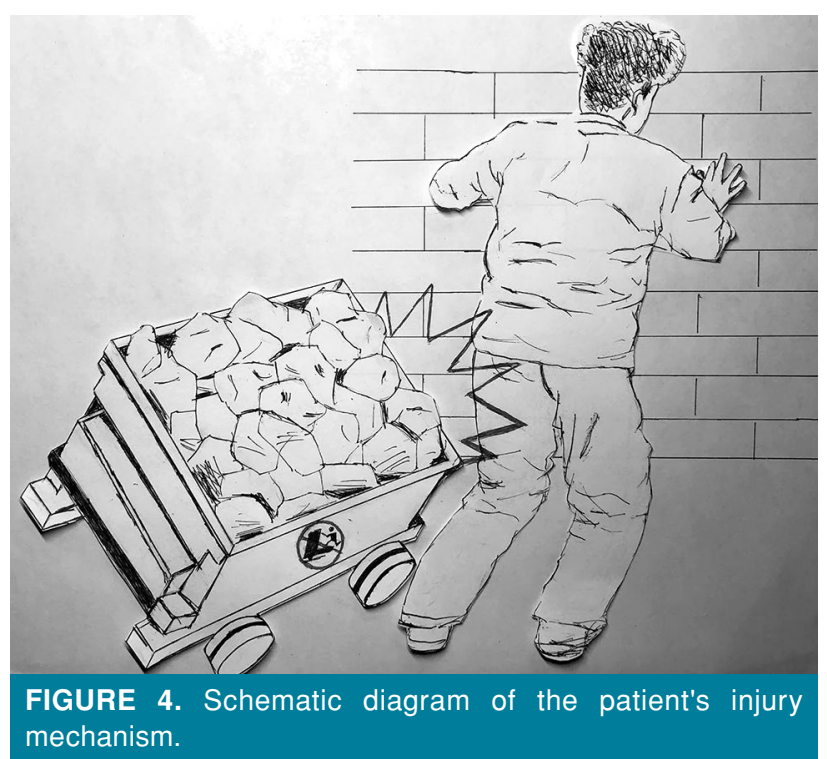


head would decrease. ${ }^{[8,10]}$ The rate of osteonecrosis varies from $5 \%$ if the hip dislocation is reduction in less than $6 \mathrm{~h}$ after injury to $50 \%$, if the hip dislocation is reduction more than $6 \mathrm{~h}$ after injury. ${ }^{[16]}$

In conclusion, given the severity of the associated complications, every effort should be made to ensure prompt diagnosis and immediate therapy. The optimal management of a patient presenting with asymmetrical hip dislocations and hemodynamic instability is disputed. A special attention must be paid to early rescue procedures, including initial circulation support and elimination of bleeding, as well as manual reduction or open reduction and internal fixation. With the application of enhanced recovery after surgery in traumatic orthopedics and the rapid coordination of various departments, the effect of hip dislocation treatment would be enhanced, and the rate of complications would decrease.

\section{Declaration of conflicting interests}

The authors declared no conflicts of interest with respect to the authorship and/or publication of this article.

\section{Funding}

The authors received no financial support for the research and/or authorship of this article.

\section{REFERENCES}

1. Agarwal S, Singh GK, Jain UK, Jyoti G. Simultaneous anterior and posterior traumatic dislocation of the hip. A case report with review of the literature. Arch Orthop Trauma Surg 2000;120:236-8.

2. Martínez AA, Gracia F, Rodrigo J. Asymmetrical bilateral traumatic hip dislocation with ipsilateral acetabular fracture. J Orthop Sci 2000;5:307-9.

3. Carpintero P, Caeiro JR, Carpintero R, Morales A, Silva S, Mesa M. Complications of hip fractures: A review. World J Orthop 2014;5:402-11.

4. Pascarella R, Fantasia R, Sangiovanni P, Maresca A, Massetti $\mathrm{D}$, Politano $\mathrm{R}$, et al. Traumatic hip fracture-dislocation:
A middle-term follow up study and a proposal of new classification system of hip joint associated injury. Injury 2019;50 Suppl 4:S11-S20.

5. Huang K, Giddins G, Zhang JF, Lu JW, Wan JM, Zhang PL, et al. Asymmetrical traumatic bilateral hip dislocations with hemodynamic instability and an unstable pelvic ring: Case report and review of literature. World J Clin Cases 2018;6:94-8.

6. Hoffman A, Taylor BC. Asymmetric bilateral hip dislocations with a pulseless left lower extremity: A case report. JBJS Case Connect 2019;9:e0479.

7. Giaretta S, Silvestri A, Momoli A, Micheloni GM. Asymmetric bilateral hip dislocation in young man: A case report. Acta Biomed 2019;90:183-6.

8. Alshammari A, Alanazi B, Almogbil I, Alfayez SM. Asymmetric bilateral traumatic hip dislocation: A case report. Ann Med Surg (Lond) 2018;32:18-21.

9. Paša L, Veselý R, Kelbl M. Bilateral asymmetric traumatic dislocation of hip joints. Acta Chir Orthop Traumatol Cech 2017;84:66-9.

10. Buckwalter J, Westerlind B, Karam M. Asymmetric bilateral hip dislocations: A case report and historical review of the literature. Iowa Orthop J 2015;35:70-91.

11. Kanojia RK, Patra SR, Gupta S. Bilateral asymmetric dislocations of hip joints: An unusual mechanism of injury. Case Rep Orthop 2013;2013:694359.

12. Pascarella R, Maresca A, Cappuccio M, Reggiani LM, Boriani S. Asymmetrical bilateral traumatic fracture dislocation of the hip: A report of two cases. Chir Organi Mov 2008;92:109-11.

13. Fang Y, Pei FX, Yang TF, Wang GL, Liu L. Traumatic asymmetrical bilateral hip dislocation: A case report and literature review. Eklem Hastalik Cerrahisi 2011;22:177-9.

14. Abdulfattah Abdullah AS, Abdelhady A, Alhammoud A. Bilateral asymmetrical hip dislocation with one side obturator intra-pelvic dislocation. Case report. Int J Surg Case Rep 2017;33:27-30.

15. Karaarslan AA, Acar N, Karci T, Sesli E. A bilateral traumatic hip obturator dislocation. Case Rep Orthop 2016;2016:3145343.

16. Phillips AM, Konchwalla A. The pathologic features and mechanism of traumatic dislocation of the hip. Clin Orthop Relat Res 2000;(377):7-10. 